\title{
Entwicklung einer miniaturisierten Multiparametersonde für limnologische Anwendungen
}

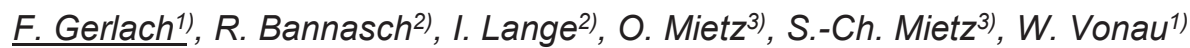 \\ 1) Kurt-Schwabe-Institut für Mess- und Sensortechnik Meinsberg e.V., Waldheim, Deutschland \\ 2) EvoLogics GmbH, Berlin, Deutschland \\ 3) Institut für angewandte Gewässerökologie $\mathrm{GmbH}$, Seddiner See, Deutschland \\ Kontakt: Frank.gerlach@ksi-meinsberg.de
}

\begin{abstract}
Einleitung
Für die Gewässerüberwachung wurde in den letzten Jahren eine Vielzahl rechtlicher Regelungen auf Länderebene und im internationalen Rahmen eingeführt. Bereits im Jahr 2000 sind mit der Europäischen Wasserrahmenrichtlinie qualitative Anforderungen für die Bewertung der Güte von Seen mit einer Fläche von mehr als 50 ha geschaffen worden. Fokus bildet hier die Begrifflichkeit eines "guten ökologischen Zustandes“. Dies beinhaltet neben einer diversen Besiedlung durch Unterwasserpflanzen und -tiere eine geringe Trophie, d.h. einen ausgewogenen, niedrigen Nährstoffgehalt, eine geringe Phytoplankton- (Chlorophyll-a) Menge ohne Massenentwicklungen sowie eine hohe Sicht-
\end{abstract} tiefe.

Die Gewässer der Industrie- und Bergbaufolgelandschaft zählen zu den künstlichen Gewässern und bilden einen eigenständigen Gewässertyp, dessen Leitbildentwicklung Inhalt zahlreicher Studien der letzten Dekaden war.

Industriefolgeseen weisen eine hohe Diversität hinsichtlich der Wasserqualität auf und hängen stark von ihrer Ursprungsgeschichte und Abbautechnologie ab. So hat das geförderte Mineralvorkommen nicht nur einen Einfluss auf den Chemismus, sondern auch auf die Morphologie des Folgesees. Auch die Art der Flutung ist für die Entwicklung des ökologischen Zustandes von großer Bedeutung.

Abhängig von der Endnutzung der Seen ergeben sich verschiedene Qualitätsansprüche, die wiederum diverse Behandlungsoptionen erforderlich machen. So muss z. B. unterschieden werden, ob der See zukünftig als Freizeitsee zum Schwimmen, Angeln oder Wassersport, als Brauchwasser oder zur landwirtschaftlichen Bewässerung genutzt werden soll. Bei einem Badegewässer stellt die toxische und hygienische Unbedenklichkeit sowie die Einhaltung der Grenzwerte hinsichtlich der EU-Badewasserrichtlinie oberste Priorität dar, während der Schutz der Biozönosen, die Naturnähe und freie Sukzession für reine Naturschutzgewässer unentbehrlich sind [1].

Derzeitig gibt es wenige kommerziell verfügbare, miniaturisierte Sensoren und Sensorsysteme für den limnologischen Bereich, insbesondere für den Flachwasserbereich bis $60 \mathrm{~m}$ Tauchtiefe, welche sich für die Applikation mit autark operierenden Wasserfahrzeugen eignen. Diese werden als routinetaugliche Messplattformen eingesetzt, um Monitoringprogramme zur Charakterisierung und Bewertung von Industriefolgeseen und darüber hinaus von Flachwasserseen auf der Basis erfasster Wasserqualitätsparameter, kosteneffizient durchzuführen.
Die im Rahmen eines europäischen FuE-Verbundvorhabens [2] zu lösende Teilaufgabenstellung bestand für das $\mathrm{KSI}$ in der Entwicklung und Realisierung einer miniaturisierten, tauchfähigen Messeinrichtung zur Bestimmung des pH-Werts, des Redoxpotenzials und der Leitfähigkeit sowie von Druck und Temperatur. Diese beinhaltet ein komplexes Messsystem mit einer miniaturisierten Multiparametersonde einschließlich der Elektronik- und Datenübertragungsmodule.

\section{Entwicklungen}

\section{Messplattform}

Die Messplattform (s. Abb.1) basiert auf einem Mehrrumpfschwimmkörper der im Projekt involvierten Fa. Evologic, Berlin. Dieser Katamaran besitzt den nötigen Auftrieb, eine ausreichende Stabilität und über einen Tender die Möglichkeit eine Winde zur Aufnahme von Tiefenprofilen zur Verfügung zu stellen.

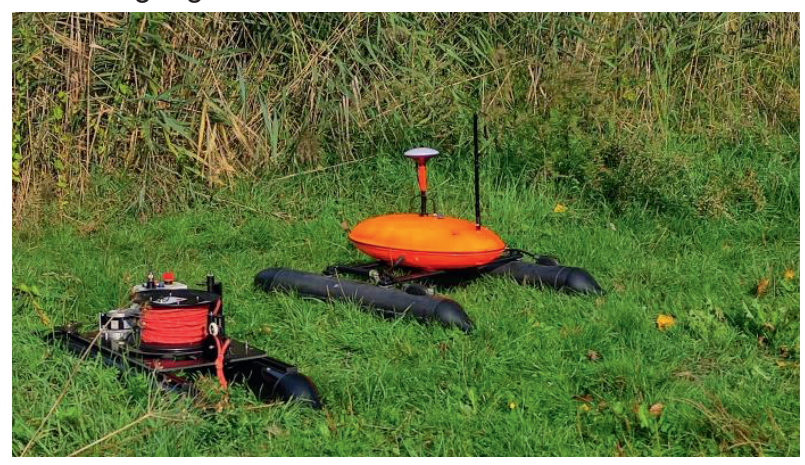

Abb. 1: Mehrrumpfschwimmkörper mit Winde zur Aufnahme der miniaturisierten Multiparametersonde

Die GPS-gestützte Navigation der Messplattform gestattet die reproduzierbare Positionierung auf Seen und in Verbindung mit der Verwendung von Echolotsensoren die Erhebung von Bathymetriedaten. Somit kann die Anordnung als effizientes Werkzeug für das Monitoring von Seen und für Kontrollmessungen nach durchgeführten Sanierungs- und Restaurationsmaßnahmen eingesetzt werden

\section{Messsystem}

Das Messsystem dient zur Erfassung, der Anzeige und dem Abspeichern von Messparametern für limnologische 
Anwendungen. Darüber hinaus ermöglicht es die Sensorkalibrierung, Signalkompensation und die eventgesteuerte Ansteuerung von Aktorik.

Das gesamte Messsystem besteht aus der Multiparametersonde, dem Funkmodul und dem Auswerterechner (Abb. 2).

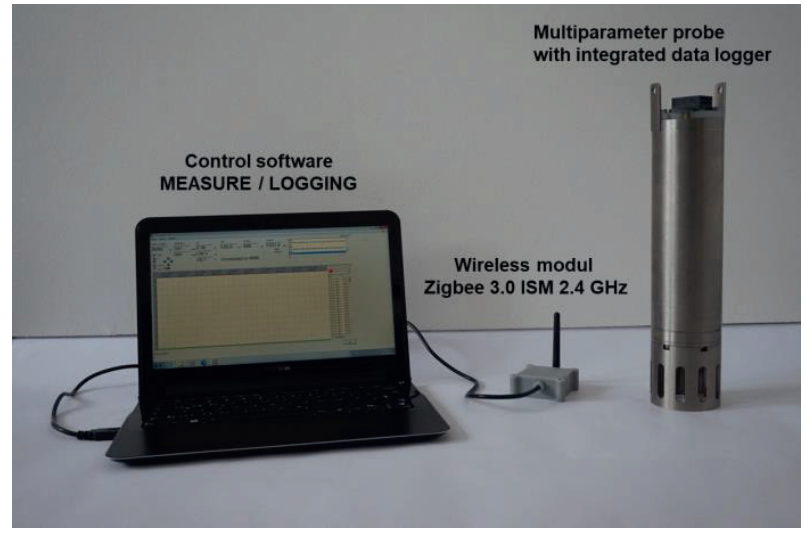

Abb. 2: Multiparameter-Messsystem mit Auswerterechner, Funkmodul ZIGBEE 3.0 und Multiparametersonde

Die miniaturisierte Sonde ist konzipiert für maximal $8 \mathrm{Ka}$ näle. Bis auf den Parameter Leitfähigkeit sind alle Kanäle galvanisch getrennt. Die Daten aller Kanäle können mit einer Abtastrate von 1 Messung/s für eine Dauer von 8 Stunden in einem internen Datenlogger abgespeichert werden. Die Multiparametersonde verfügt über eine Echtzeituhr (RTC). Somit sind alle Datensätze mit einem Zeitstempel für Synchronisierungszwecke versehen.

Die Stromversorgung wird über einen 3,7 V LithiumionenAkku mit 3500 mAh und einer implementierten Schutzschaltung sichergestellt. Diese ist für einen maximalen Entladestrom von 10 A ausgelegt. Eine Ladeschaltung ist ebenfalls implementiert. Die Ladung erfolgt über ein konventionelles USB-Ladegerät und beträgt nach 6 Stunden $2400 \mathrm{mAh}$.

Die Verbindung vom Steuerrechner zum System wird über ein DIGI XBEE 3 ZIGBEE 3.0 Funkmodul hergestellt. Das Funkmodul arbeitet im 2,4 GHz-Bereich und hat einen Sende-/Empfangs-Stromverbrauch von 40/17 mA.

Die maximale Sichtentfernung beträgt mit der momentan intern verbauten Antenne ca. $25 \mathrm{~m}$. Dies gestattet das Starten und Konfigurieren sowie die Messwertverfolgung des Messsystems im ufernahen Bereich. Werden die Anforderungsprofile in Zukunft geändert, so sind mit einer geänderten Elektronik und einer extern angeordneten Antenne überbrückbare Sichtentfernungen bis $1000 \mathrm{~m}$ möglich.

\section{Sensorkonfiguration}

Der Innovationsgehalt des Forschungsgegenstandes begründet sich zu einem wesentlichen Teil aus der Verwendung von miniaturisierten Sensoren zur Detektion der Wasserparameter. Vorab durchgeführte Forschungsaktivitäten $[3,4]$ bilden die wissenschaftliche und technologische Ba- sis für die Entwicklung einer miniaturisierten Sensorkonfiguration für autonome Fahrzeuge. Hierfür kamen verschiedene Fertigungstechnologien zur Anwendung (Abb. 3).

Der pH-Wert wird aus Genauigkeitsgründen mit einer miniaturisierten $\mathrm{pH}$-Elektrode in konventioneller Bauart gemessen. Für die Leitfähigkeitsmesszelle und die Redoxelektrode kommen Systeme in Dickschichttechnik zum Einsatz. Das für die potentiometrischen Parameter notwendige Referenzsystem besteht im Kern aus einer druckstabilen, polymerbasierten Metall/Metalloxidelektrode mit Reservoir. Dieses System verfügt über eine für die Applikation ausgesprochen gute Langzeitstabilität bei einer geringen Gefahr von Vergiftungserscheinungen.

Ergänzt wird das Sensorsetup durch umschaltbare Drucksensoren wahlweise 3/30 bar oder 30/300 bar. Dies gewährleistet eine hohe Auflösung der Tauchtiefe, auch im Flachwasserbereich. Der zentral angeordnete schnelle Temperaturfühler ermöglicht die effektive Temperaturkompensation der Sensorsignale.

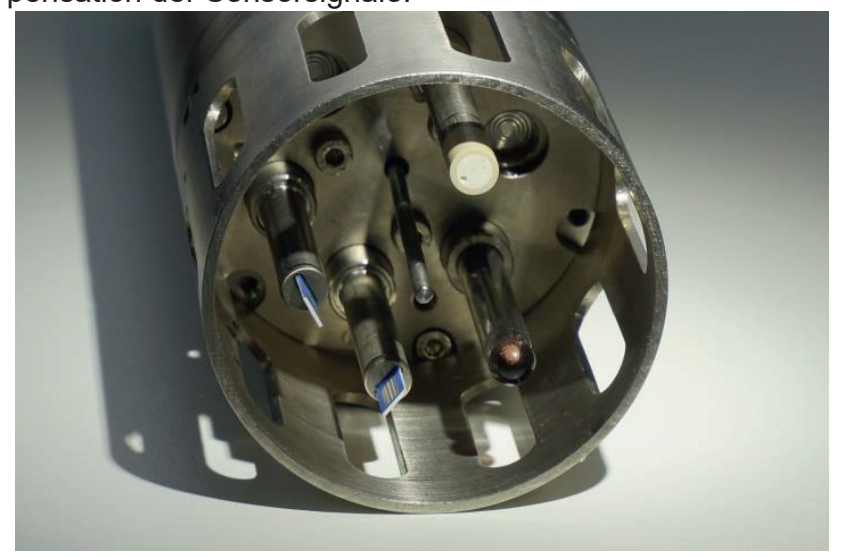

Abb. 3: Miniaturisierte Sensorkonfiguration ( $\mathrm{pH}-\mathrm{Gla}-$ selektrode, 4-Elektroden-Leitfähigkeitszelle, Pt-Redoxpotenzial-Elektrode, polymerbasierte Referenzelektrode, Drucksensor und Temperatursensor)

Der Einsatz o.g. Technologien für die Herstellung und die Umsetzung von All-Solid-State-Prinzipien ermöglicht in geeigneter Weise die konsequente Miniaturisierung aller zur Anwendung kommenden Sensoren. Demgegenüber stehen die sensorspezifischen Nachteile, die durch die geänderten Konstruktionen, verminderten Reservoirs und verminderten Elektrodenflächen auftreten bzw. verschärft werden. Im Folgenden soll diese Problematik am Beispiel der planaren 4-Elektroden-Leitfähigkeitszelle diskutiert werden.

\section{Planare 4-Elektroden-Leitfähigkeitsmesszelle}

Diese Sensoren werden mittels Siebdruck auf planare Aluminiumoxid-Substrate (Dicke: 0,63 mm) gedruckt. Die Elektroden bestehen aus Platin und verfügen, in Folge einer glasfreien Pastenformulierung, nach dem Sintervorgang über eine poröse Morphologie mit einer hohen spezifischen Oberfläche (Abb. 3). 


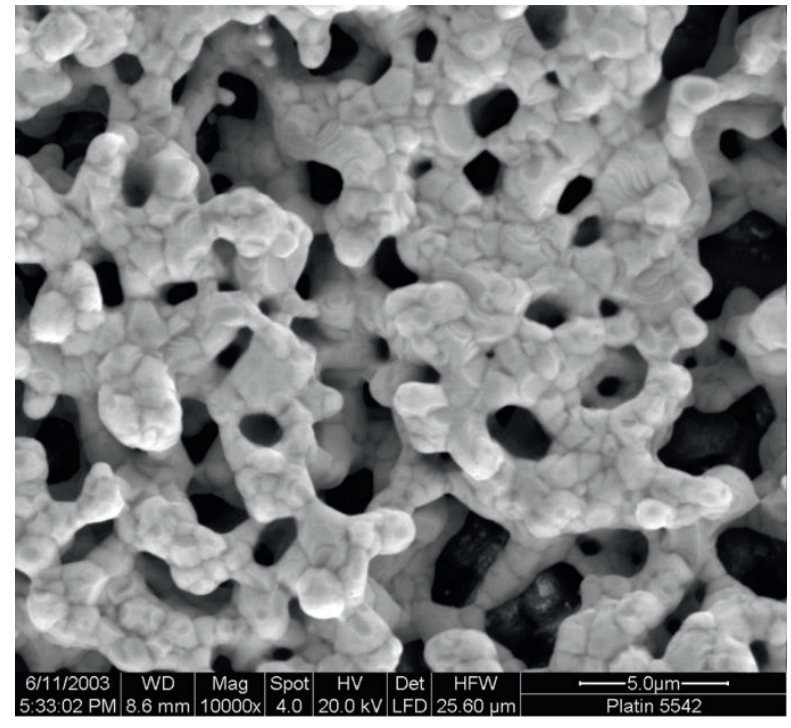

Abb. 3: poröse Platinelektrode aus glasfreier Pastenformulierung gesintert

Die Abb. 4 zeigt das realisierte Sensorlayout. Dabei besitzen die äußeren, stromführenden Elektroden eine effektive Länge von 7,9 mm und einer Breite von 0,72 $\mathrm{mm}$ und somit eine Fläche von ca. $5,7 \mathrm{~mm}^{2}$. Die inneren potenzialabgreifenden Elektroden sind ebenfalls 7,9 mm lang und besitzen eine Breite von ca. 0,5 mm mit einem Elektrodenabstand von ca. $350 \mu \mathrm{m}$. In dieser Konstellation ergibt sich eine Zellkonstante von ca. $0,5 \mathrm{~cm}^{-1}$. Damit ist der Messbereich, der am häufigsten in der natürlichen Umwelt auftretenden Leitfähigkeiten mit einer geringen Fehlerbandbreite abgedeckt.

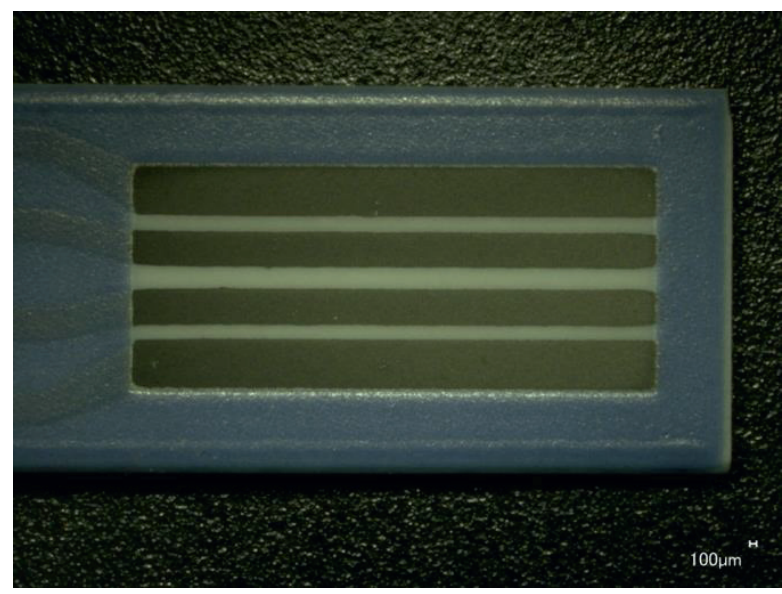

Abb. 4: Sensorlayout der 4-Elektroden-Leitfähigkeitszelle

\section{Ergebnisse}

Vorangestellte Impedanzspektren (Abb. 5) zur Bestimmung der optimalen Generatorfrequenz der Elektrodenanordnung bei einer Amplitude von 50 mV ergaben, dass sich der Phasenwinkel über dem gesamten Frequenzbereich von $1 \mathrm{~Hz}$ bis $100 \mathrm{kHz}$ nahe $0^{\circ}$ bewegt. Dies gilt auch für den gesamten betrachteten Temperaturbereich von $5{ }^{\circ} \mathrm{C}$ bis $25^{\circ} \mathrm{C}$. Damit wurde die Messfrequenz auf $500 \mathrm{~Hz}$ festgelegt, um die Belastungen der Elektroden durch Polarisationseffekte gering zu halten.

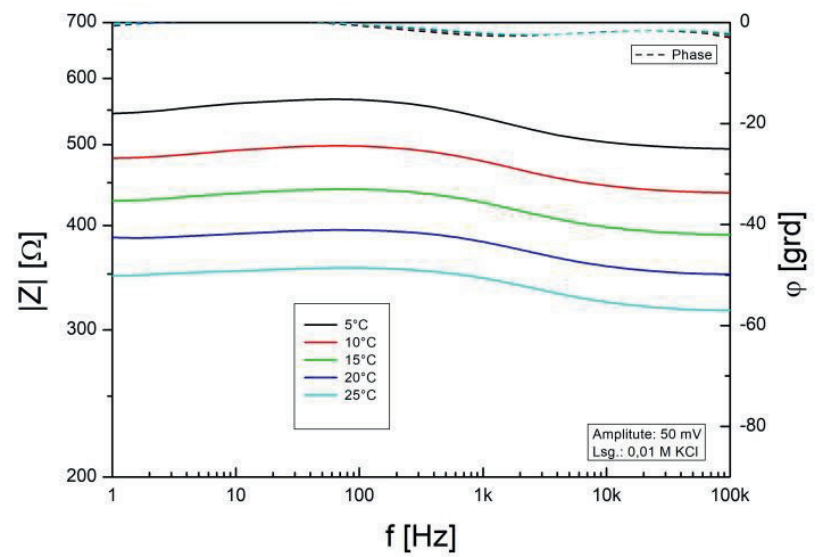

Abb. 5: Impedanzspektren der Anordung (50 mV, Lösung $0,01 \mathrm{M} \mathrm{KCl}$, Temperaturbereich $5^{\circ} \mathrm{C}-25^{\circ} \mathrm{C}$ )

Messungen der Anordnung in potenziostatischer Betriebsweise bis zu Lösungskonzentrationen von $0,02 \mathrm{M} \mathrm{KCl}$ zeigen sehr stabile Verhältnisse im betrachteten Temperaturbereich. Erst ab einer Konzentration von 0,1 M KCl kam es zu Unregelmäßigkeiten sowohl in Betrag und Phase der Kurve, begründet durch Gasblasenentwicklungen an den Elektroden.

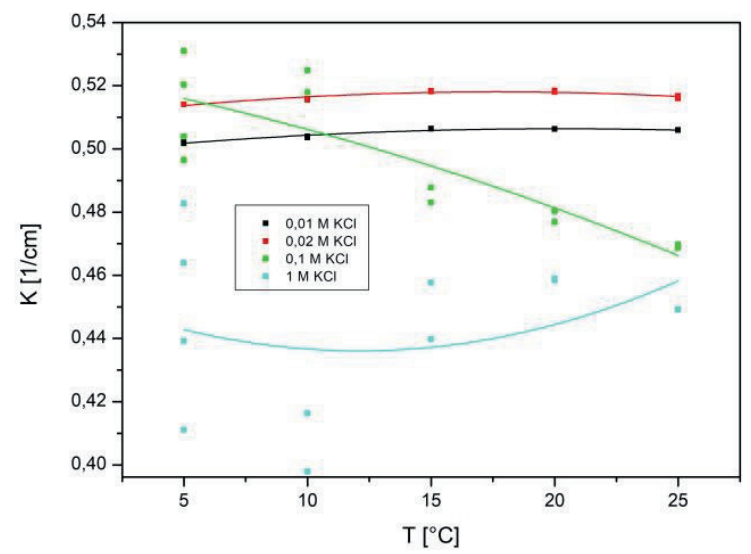

Abb. 6: Zellkonstante als Funktion der Temperatur und der Konzentration der Lösung (potenziostatische Betriebsweise, Spannung: $30 \mathrm{mV}$, Frequenz $500 \mathrm{~Hz}$ )

Der entscheidende Parameter bei der Anwendung von Leitfähigkeitszellen ist die Zellkonstante K. Diese stellt sich vorzugsweise bei Zweielektrodenmesszellen als Quotient der geometrischen Größen Elektrodenabstand und Elektrodenfläche oder aber als Produkt des gemessenen Widerstandes der Anordnung und der spezifischen Leitfähigkeit 
der Messlösung dar. Diese Zellkonstante (Abb. 6) und deren Konstanz bestimmt in Verbindung mit der angeschlossenen Elektronik erheblich den verwertbaren Messbereich und die Reproduzierbarkeit der Messung.

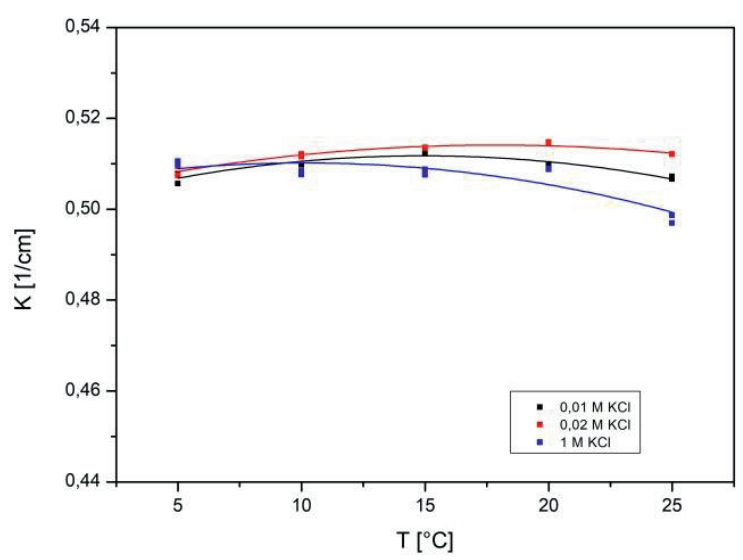

Abb. 7: Zellkonstante als Funktion der Temperatur und der Konzentration der Lösung, (galvanostatische Betriebsweise, Strom $50 \mu \mathrm{A}$, Frequenz $500 \mathrm{~Hz}$ )

Eine Möglichkeit, die Performance der Messzelle noch weiter zu verbessern, ist das Betreiben der Messzelle im galvanostatischen Mode (Abb. 7). Dabei wurde im vorliegenden Fall der treibende Strom an den äußeren Elektroden auf $50 \mu \mathrm{A}$ eingestellt.

Die signifikant unterschiedliche Auffächerung der Kurvenschar in Folge der Abhängigkeit der Zellkonstante von der Konzentration der Messlösung und der Temperatur ist auf die planare Bauform zurückzuführen. Da es sich um Streufeldsensoren handelt, deren sphärische Feldlinienausbildung u.a. von äußeren Bedingungen abhängig sind. Dem könnte mit zusätzlichen Abschirmelektroden, wie sie bei hochpräzisen Sensoren in der Tiefseeforschung verwendet werden, entgegengewirkt werden.

Ein weiterer Punkt zur Fehlerminimierung bei der Leitfähigkeitsmessung in natürlicher Umgebung ist die entsprechende Temperaturkompensation. Üblich ist die Kompensation entsprechend der Norm DIN EN 27888 [5], die einen Vorschlag zur Temperaturkompensation in „natürlichen Wässern“ enthält. Dieses Verfahren ist bei einer stark ausgeprägten Schichtung aerober und anaerober Zonen des Wasserkörpers in Verbindung mit einhergehenden Rücklöseprozessen aus dem Sediment nur bedingt anwendbar. Einen Ausweg bietet hier die Probennahme mit nachfolgender Bestimmung des spezifischen Temperaturkoeffizienten des Gewässers.
Bei dem Parameter pH-Wert kann auf die herkömmliche Zweipunktkalibrierung unter Anwendung des NERNSTFaktors zur Temperaturkompensation bzw. bei der Ermittlung des Redoxpotenzials die zusätzliche Angabe der Messtemperatur zurückgegriffen werden. Hervorzuheben ist darüber hinaus, dass sich die verwendete All-SolidState-Referenzelektrode auf polymerer Basis als überaus stabil erwies und alle Labor- und Feldmessungen mit einem Exemplar durchgeführt werden konnten, welches danach zum Ausgangszustand nur wenige $\mathrm{mV}$ Potenzialunterschied aufwies, obwohl diese sowohl unterschiedlichen Temperatur- und Druckbedingungen als auch dem Kontakt mit Bodenschlamm und schwefelwasserstoffhaltigen Medien ausgesetzt war. Für weitergehende limnologische Untersuchungen im Zusammenhang mit Restaurierungs- und Sanierungsfragestellungen wäre es wünschenswert, einen miniaturisierten optischen Sauerstoffsensor zu implementieren.

\section{Auswertung}

Die von der Multiparametersonde detektierten Werte (Abb. 8) sind Teil der Basisparameter, die für die Integration in das gleichzeitig entwickelte Softwaremodul notwendig sind und das komplexe Bild der Gewässergüte abbilden sollen.

Darin stellt der $\mathrm{pH}-$ Wert einen maßgeblichen Parameter in Industriefolgeseen dar, da die Freisetzungsraten der meisten Metalle und Halbmetalle stark $\mathrm{pH}$-abhängig sind und darüber hinaus die meisten Wasserlebewesen einen relativ engen $\mathrm{pH}$-Toleranzbereich besitzen.

Weitere Inputparameter, die die Trophieentwicklung und Wahl geeigneter Entwicklungsmöglichkeiten für Industriefolgeseen beeinflussen, sind z. B. die Hauptnährstoffe auf Basis von Phosphor-, Stickstoff- und Kohlenverbindungen sowie das Vorhandensein externer oder interner Belastungsquellen. Zusätzlich zu chemischen/physikalischen und biologischen Faktoren sind weiterhin besondere hydraulische (z. B. Wasserstandsschwankungen) und morphometrische Randbedingungen zu betrachten. Dabei beeinflusst das Verhältnis von Hypo- zu Epilimnion sowie die Ufersteilheit und die daraus resultierende Stabilität z. B. die Habitatqualität und damit die Ansiedlung von aquatischen Makrophyten.

Je nach Folgenutzung und Belastungsart ergeben sich im Softwaremodul Empfehlungen für Restaurations- und Sanierungsverfahren, wie z. B. die Flutung mit Fremdwasser, Maßnahmen im Einzugsgebiet, biogene und chemische Abdichtungen des Sediments um Rücklösungsprozesse zu beeinflussen, Kalkung zur Neutralisation, biogene Sulfatund Eisenreduktionen, kontrollierte Eutrophierung etc. [1]. 


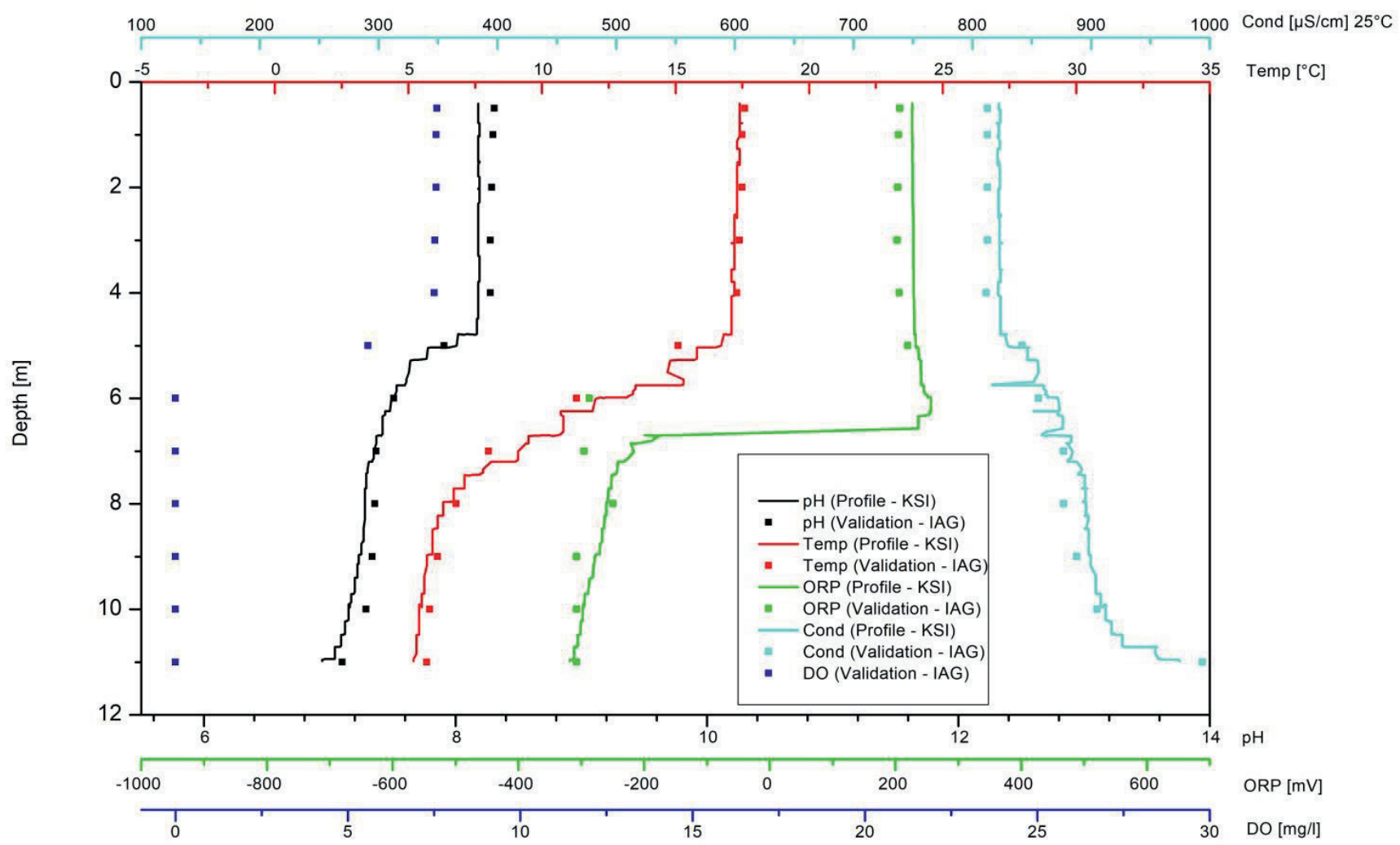

Abb. 8: Tiefenprofilmessungen mit Multiparameter-Messsystem (Kurt-Schwabe-Institut) und Referenzmessungen mit konventioneller Messtechnik (Institut für angewandte Gewässerökologie)

\section{Zusammenfassung}

Die im vorliegenden Beitrag vorgestellten miniaturisierten Sensoren zur Bestimmung von Wasserqualitätsparametern, wie z.B. pH-Wert, Redoxpotenzial und elektrische Leitfähigkeit sind in Verbindung mit den erfassten Parametern Druck und Temperatur dazu geeignet, kleine autonome Wasserfahrzeuge sogenannte (ASV - Autonomous Surface Vehicle) sensorisch auszurüsten, um komplexe limnologische Problemstellungen effektiv und kostengünstig zu bearbeiten. Es konnte nachgewiesen werden, dass diese miniaturisierten Sensoren, welche mit neuen innovativen Fertigungstechnologien hergestellt wurden, mit Referenzmessungen traditioneller Messtechnik vergleichbare Werte liefern. Die Kombination von GPS basierter Navigation, der Erstellung einer Bathymetrie und die Erfassung reproduzierbarer Tiefenprofile eröffnet die Möglichkeit der objektorientierten Modellierung von Wasserkörpern, die Ableitung effektiver Sanierungskonzepte und das nachfolgende Monitoring der Wasserqualitätsparameter.

\section{Literatur}

[1] Nixdorf, B.; Mutz, M.; Wiegleb, G.: Die Bewertung von Tagebaugewässern und ihrer Entwicklung im Spiegel ökologischer und wasserwirtschaftlicher Rahmenbedingungen. Kapitel 8. In: Kapfer, M. und Nixdorf, B. (Hg.): Gewässerreport (Nr. 5): Methoden der limnologischen
Untersuchung und Bewertung von Stand und Fließgewässern. Cottbus (Aktuelle Reihe, 1/99), 1999.

[2] Eurostars Verbundvorhaben „Autark funktionsfähiges mobiles Messsystem für das Monitoring der Wasserqualität in Industriefolgeseen - HydroSense“

(FKZ: 01QE1907C)

[3] Schindler, W.; Gerlach, F.; Kaden, H.: pH-Sensor für Präzisionsmessungen in Tiefenwässern. Sensormagazin (1995) 3, 6-9

[4] Gerlach, F.; Berthold, M.; Vonau, W.: Chemosensorik für die Erkundung von Tiefenbohrungen. Geothermiekongress, ISBN-13-978- 932570-69-8, Essen, 11.13.11.2014

[5] DIN EN 27888: Bestimmung der elektrischen Leitfähigkeit (ISO 7888:1985). DIN Deutsches Institut für Normung e.V. Berlin

\section{Danksagung}

Das Projekt „HydroSense“ (FKZ: 01QE1907C) wird im Rahmen des europäischen Förderprogramms „Eurostars“ durchgeführt und die deutschen Partner aus Mitteln des Bundesministeriums für Bildung und Forschung gefördert.

Das Kurt-Schwabe-Institut für Mess- und Sensortechnik Meinsberg e.V. wird durch Steuermittel auf der Grundlage des vom Sächsischen Landtag beschlossenen Haushaltes mitfinanziert. 